\title{
Linear Isomorphic Regulators
}

\author{
Vladimir Kulabukhov ${ }^{1, *}$ \\ Uspensky Avionica Moscow Research and Production Complex, Moscow, Russia
}

\begin{abstract}
A simple formalized procedure of the synthesis of isomorphic regulators for linear objects "single input - single output". Shown that regulators are isomorphic multiplicative and have the ability to "limit" in terms of quality of transition processes in comparison with additive regulators of the traditional structure.
\end{abstract}

\section{Introduction}

The analysis shows [1] that the modern methods of synthesis of regulators are not enough formalized. This applies even to simple linear systems "single input single output", which are often used in practice. To ensure high quality of transition processes in control loops, such systems need to involve heuristic (artificial) techniques. In order to search for a common solution to this problem, apparatus of modern algebra [2, 3, 4] is frequently used. In this paper [4], based on common algebraic principle of the isomorphy in the theory of systems, the concept of controllability with accuracy up to some isomorphic model is introduced. Proved in [4], the theorems have formally allowed to define the structure of regulators for general systems and linear systems. In this article, the method of synthesis of a new type of regulators - isomorphic controllers - is considered based on the results of Article [4], for linear systems of the type "single input - single output". Unlike traditional additive of regulators, isomorphic regulators have multiplicative form. An example of the synthesis of isomorphic controller for linear object is presented. Here are shown the advantages of isomorphic regulators, which include the formal rules of the synthesis and ensuring the maximum achievable quality of transition processes in comparison to traditional regulators.

\section{Linear isomorphic regulators}

The following system is considered,

$$
\dot{X}=A(t) X+B(t) U, X \subset R^{n}, U \subset R^{m},
$$

which can be described in Laplace form with zero initial conditions has the form:

$$
X(p)=(p I-A)^{-1} B U(p)=\Phi(p) Y(p),
$$

where $\Phi(p)=(p I-A(p))^{-1}-$ fundamental (transition) of the system matrix (1) in the Laplace form, $I$ - identity matrix, $Y(p)=B(p) U(p)$ - some variable that characterizes the internal state of the system. Matrix $\Phi(p)$ for linear systems is always the opposite, i.e. an isomorphism. The Laplace form of system (1) is converted from the additive to the multiplicative form.

$$
\left\{\begin{array}{l}
Y(p)=B U(p), \\
X(p)=\Phi(p) Y(p) .
\end{array}\right.
$$

From [4], that's needed for mappings of matrices from equation (2), commutative diagram has the form and it's shown in Fig. 1.

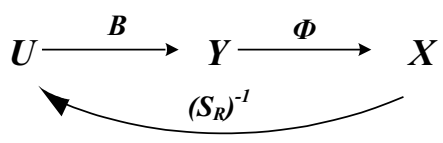

Fig. 1. The composition of mappings to determine the controllability of linear systems.

After Fig. 1, for a linear object (1) in the multiplicative form (2) isomorphic with accuracy up to isomorphism $\Phi$ regulator $S_{R}^{-1}$ will have the form:

$$
S_{R}^{-1}=B^{-1} \Phi^{-1}(p)=B^{-1}(p I-A(p)) .
$$

Those regulators, unlike known additive regulators that use the addition-subtraction operation will be called isomorphic multiplicative regulators. Conditions of controllability of object (2) and the rules for determining the inverse matrices $S_{R}^{-1}$ and $B^{-1}$ with accuracy up to isomorphism $\Phi$ are defined in [4]. It is shown [4] that for a controlled object within the commutative diagram, there are always inverse matrix $S_{R}^{-1}$ and $B^{-1}$, even if the original matrices are rectangular and out of irreversible chart. Suppose that the system is controllable. Require that the actual value of the vector $\mathrm{X}$ tracked a desired value $X_{\varkappa}$ in accordance with the movement of the reference model with a transfer function $W_{э м}$. Those problems of synthesis of the prototype have universal character [2]. Reference model is selected, for example, taking into account the quality of engineering criteria. 
Considering various forms of reference model, the equation (1) of the control system take the form:

$$
\begin{gathered}
\dot{X}=A X+B U, X_{0} \\
\dot{X}_{3}=C X_{3}+D X_{\varkappa}, X_{30}=X_{0}, \\
X=I X_{3}=I X_{\mathscr{W}},
\end{gathered}
$$

where $X_{3}$ - "instantaneous" set value, which must exactly match the output X. Reference model equations (5) and (6) correspond to the transfer functions $W_{э м}=(p I-C)^{-1} D$ и $W_{э м}=I$, where $I$ - identity matrix.

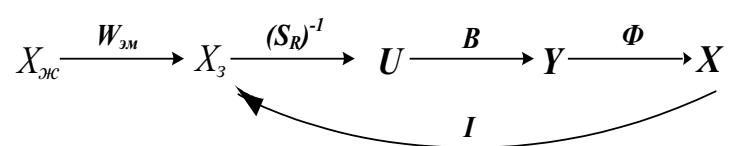

Fig. 2. The composition of mappings for tracking the reference model.

In Fig. 2 it is presented a commutative diagram, obtained from Fig. 1, taking into account (4) - (6). For Fig. 2, equation (3) takes the form;

$S_{R}^{-1}=B^{-1}(p) \Phi^{-1}(p) I=B^{-1}(p) \Phi^{-1}(p)=B^{-1}(p)(p I-A(p))$,

$S_{R}=\Phi(p) B(p)$,

where $S_{R}^{-1}$ and $B^{-1}$ must satisfy conditions [4]:

$I_{x}^{\text {right }}=S_{R} S_{R}^{-1}, I_{x}^{\text {left }}=\Phi \Phi^{-1}, I_{x}^{\text {left }}=I_{x}^{\text {right }}=I$,

$I_{U}^{\text {left }}=S_{R}^{-1} S_{R}, I_{U}{ }^{\text {right }}=B^{-1} B, I_{U}{ }^{\text {left }}=I_{U}{ }^{\text {right }}$.

Here $I_{x}^{\text {left }}, I_{x}^{\text {right }}$ - left and right unit on the set of $X_{3}=X, I_{U}^{\text {left }}$ and $I_{U}{ }^{\text {right }}$ - left and right units to the U. In this case, unit on $X_{3}$ is equal to the identity matrix $I$. In general, when $\mathrm{m} \geq \mathrm{n} \quad I_{U}^{\text {left }}$ and $I_{U}{ }^{\text {right }}$ can be non-unit matrices in the usual sense, but such that:

$$
I_{U}^{\text {left }}=I_{U}{ }^{\text {right }}=I_{U}=\left(I_{U}\right)^{-1} .
$$

Equations (7) and (8) allow to determine an isomorphic regulator $S_{R}^{-1}$. If the model (4) exactly matches the object, the control loop with a regulator $S_{R}^{-1}$ as feedback (FB) which is shown in Fig. 2, acquires a "linear" form (see. Figure 3) without the "cycles" (without visible FB).

$$
X_{\mathscr{H}} \stackrel{\boldsymbol{W}_{э M}}{\longrightarrow} X_{3} \stackrel{\left(S_{R}\right)^{-1}}{\longrightarrow} \mathrm{U} \stackrel{\boldsymbol{B}}{\longrightarrow} \boldsymbol{Y} \stackrel{\boldsymbol{\Phi}}{\longrightarrow} X
$$

Fig. 3. "Linear" loop with the isomorphic multiplicative regulator.

$$
X_{\mathscr{H}} \stackrel{\boldsymbol{W}_{э M}}{\longrightarrow} X_{3} \stackrel{\left(S_{R}\right)^{-1}}{\longrightarrow} U \stackrel{S_{R}}{\longrightarrow} X
$$

Fig. 4. "linear" loop with maximum achievable control quality.

$$
X_{\varkappa} \stackrel{\boldsymbol{W}_{\text {эм }}}{\longrightarrow} X_{3} \stackrel{\left(\boldsymbol{S}_{R}\right)^{-1}}{\longrightarrow} U \stackrel{\text { Объект }}{\longrightarrow} X
$$

Fig. 5. "Linear" object control loop
It follows from (7) and Fig. 3, the circuit considering equation $S_{R}=\Phi(p) B(p)$ is converted to the form which is shown in Fig. 4. This "linear" loop realizes condition $S_{R} S_{R}{ }^{-1}=I$ of accurate tracking $X=X_{3} \quad X=X_{3}$ at any time. "Linear" loop does not mean that there is no FB from $X$ to $U$. The presence and structure of FB "encrypted" in the regulator $S_{R}^{-1}$. Unlike traditional additive regulators summators in control loops, a regulator SR-1 is multiplicative; such regulator requires only multiplication of matrices present in the "linear" loop. As can be seen from Fig. 4, object movement with the right synthesized isomorphic to control completely and with absolute accuracy is determined by the movement of the reference model with the transfer function $W_{\ni м}$. In this sense, the isomorphic regulator is limiting by the quality of transition processes. Since every object has a dynamic and limited power controls, as the preferred option should be considered reference model (5) from $W_{\ni M}=(p I-$ $C)^{-1} D$. Thus, $X_{\mathscr{N}}$ may comprise only a few components of $X$. On the other components of $X$ and all components of $U$ may be restricted. Let's consider the case without restrictions.

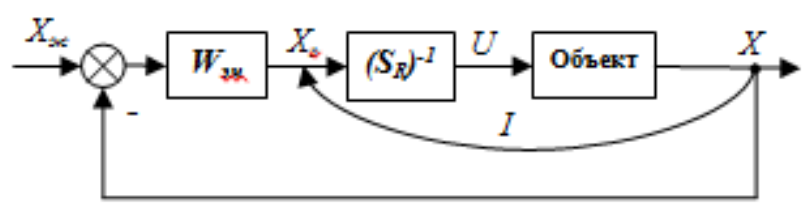

Fig. 6. The robust control loop.

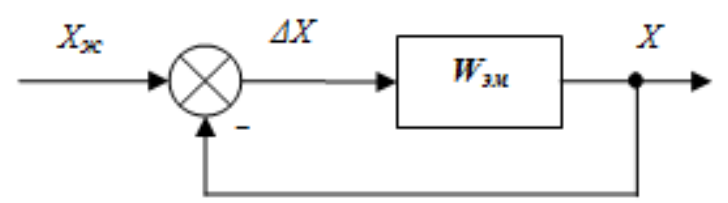

Fig. 7. The robust control loop model with adequate object.

If the objects are controlled and it's obtained "linear" loop shown in Fig. 3-5, it is necessary to check out through modeling it on the robustness, by asking variations of parameters of matrixes $\Phi$ and $B$. If the result is satisfactory, the loop can be used for control. If the object is significantly different from the model (4) used in the synthesis of the regulator, the "linear" loop can be insufficiently robust. Then it is necessary to cover it with additional FB, as shown in Fig. 6. For the adequate model object (4), transfer function "control object" from $X_{3}$ of $X$ close to the unit $W_{P O} \approx I$ and a closed loop of $X_{\varkappa}$ to $X$ (see. Fig. 6) will be as shown in Fig. 7, with the transfer function $W=W_{э м}\left(I+W_{э м}\right)^{-1}$. That is, the characteristics of a robust control loop, as before, will be determined only by the structure of reference model $W_{э M}=(p I-C)^{-1} D$. Next, let's consider an object with one input and one output. In the scalar case, at $W_{\ni M}=k_{M}\left(T_{M} p+1\right)^{-1}$, the transfer function of the closed loop will be obtained.

$$
W=\frac{k_{M}}{T_{M} p+1+k_{M}}=\frac{k_{M}}{\left(1+k_{M}\right)\left[\frac{T_{M}}{\left(1+k_{M}\right)} p+1\right]} \approx \frac{1}{T_{M}^{*} p+1}
$$


Where, $T_{M}^{*}=\frac{T_{M}}{\left(1+k_{M}\right)}$, which provides astatism on controlled coordinates at sufficiently large $k_{M}$. At the same time $T_{M}^{*}$ is $\left(1+k_{M}\right)$ times less than $T_{M}$ and the transition process in the robust loop in comparison with the "linear" loop is sharply reduced, which could adversely affect the stability and cost controls. To ensure the prior time of transition process in robust loop simultaneously with increment $k_{M}$, it's necessary to increase $\left(1+k_{M}\right)$ times and time constant $T_{M}$ to $W_{\ni M}$ times, which has the form $W_{\text {ЭM }}=k_{M}\left(T_{M}\left(1+k_{M}\right) p+1\right)^{-1}$, where $T_{M}$ - the prior time constant (before the introduction of additional feedback and increase). Then, the transfer function of the closed loop shown in Fig. 7, takes the form:

$$
W=\frac{k_{M}}{T_{M}\left(1+k_{M}\right) p+1+k_{M}}=\frac{k_{M}}{\left(1+k_{M}\right)\left(T_{M} p+1\right)} \approx \frac{1}{T_{M} p+1}
$$

and at large $k_{M}$ will practically match exactly the transfer function of the original loop without additional FB.

\section{Example}

Let's consider the specific view of matrices $A$ and $B$ in the object model (4). Let

$$
A=\left[\begin{array}{ll}
a_{11} & a_{12} \\
a_{21} & a_{22}
\end{array}\right] \text { and } B=\left[\begin{array}{cc}
b_{11} & 0 \\
0 & 0
\end{array}\right] .
$$

Equations (4) and (11) can be described in the scalar form as follow:

$$
\left\{\begin{array}{l}
\dot{x}_{1}=a_{11} x_{1}+a_{12} x_{2}+b_{11} u_{1}, \\
\dot{x}_{2}=a_{21} x_{1}+a_{22} x_{2} .
\end{array}\right.
$$

By considering $x_{2}=a_{21}\left(p-a_{22}\right)^{-1} x_{1}=a x_{1}$, where $a=a_{21}\left(p-a_{22}\right)^{-1}$, and equation (12) could be transformed in to the form:

$$
\left\{\begin{array}{l}
x_{1}=b u_{1} \\
x_{2}=a x_{1}
\end{array}\right.
$$

where $b=\frac{b_{11}}{p-a_{11}-a_{12} a}$.

For (4), by considering (11) in the form (13) diagram is shown in Fig. 3. In this case, $B=b, \Phi=a$. The transfer function of control object $W_{O}$ has the form:

$$
W_{O}=s_{r}=a b=\frac{b_{11} a_{21}}{p^{2}-\left(a_{11}+a_{22}\right) p+\left(a_{11} a_{22}-a_{12} a_{21}\right)}
$$

If the transfer function of object is known, then it is possible to obtain "linear" control loop from Fig (5) as shown in Fig (8).

$$
X_{\mathscr{H}} \stackrel{\boldsymbol{W}_{э, \mu}}{\longrightarrow} X_{3} \stackrel{\left(\boldsymbol{S}_{R}\right)^{-1}}{\longrightarrow} \mathrm{U} \stackrel{\boldsymbol{W}_{\boldsymbol{O}}}{\longrightarrow} X
$$

Fig. 8. "linear" loop for the object (13).

From Fig. 8, it implies in the scalar case, for the object of the "single input - single output" regulator definition reduces to the handling of the transfer function of the object, that is,

$$
S_{R}^{-1}=W_{O}^{-1} .
$$

Let the elements of the matrices (11) have the following values:

$$
A=\left[\begin{array}{cc}
-4 & -1,14 \\
1 & -0,62
\end{array}\right] \text { and } B=\left[\begin{array}{cc}
-7,5 & 0 \\
0 & 0
\end{array}\right] \text {. }
$$

Simulation of an ideal loop (see in Figure 8) with the regulator (15) and the type of reference model $W_{\text {Эм }}=k(T p+1)^{-1}$ at $k=1$ and $T=0,4$, it showed that $x_{2}$ tracks accurately given reference model movement. At inaccurate knowledge of elements $a_{i j}$ in (16), in the "linear" loop, working off error $x_{2}$ of error order of work $a_{i j}$ appears. Fig (9) shows the transition process (upper graph) at $\mathrm{a}_{21}=1.25 \quad(+25 \%)$. Short loop of additional operating circuit according to Fig. 6-7 gives the loop robustness property. Fig (9) (lower graphic) shows that in the loop with the additional FB at the increased gain factor to a value of $\mathrm{k}=99$ with a simultaneous increased $\mathrm{T}$ in 1 times, according to (10) even at the parameter a 21 is increased to $25 \%$, the quality of transition process remains high. Similar results were obtained at varying other matrix elements (16).

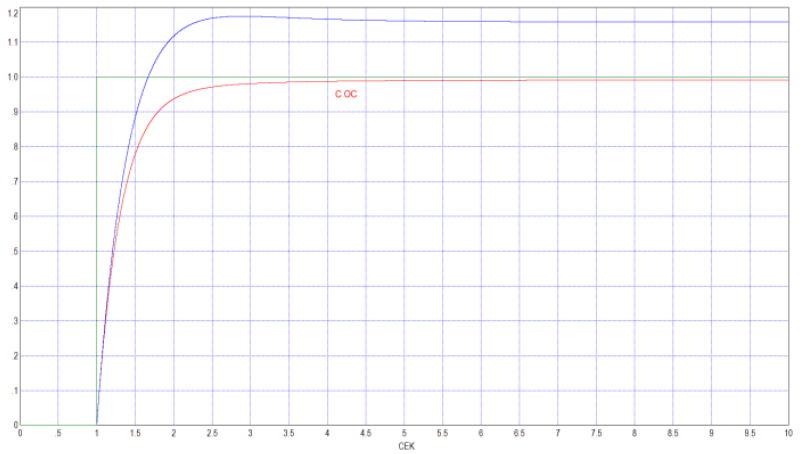

Fig. 9. Simulation of control loops by object (16), (4) at varying $a_{i j}$.

The simulation showed that the "linear" circuit has suitable robustness and for circuit with additional feedback, range of variation of matrix elements (16), while maintaining the quality of transients can be tens percent. Thus, loop of astatism can be controlled by changing gain factor $k$. 


\section{Conclusion}

Synthesis rules of isomorphic regulators on the basis of the theory [4] are examined. It is shown that the isomorphic regulators are multiplicative, and the procedure of their synthesis for the object with one input and one output has been formalized as much as possible: synthesis of regulator is reduced to handling of the transfer function of the object. In this case, the required quality of transition processes is provided by selecting of the reference model. In this sense, isomorphic regulators have the property of "limit" on the level of achievable quality of transition processes in comparison with known regulators. An example of the synthesis and simulation of isomorphic regulator showed this dignity.

\section{References}

1. V. Bulgakov, V. Kulabuhov. Instruments (Pribory), 1 (151), 39-44 (2013)

2. V. Bukov, Attachment systems. Analytical approach to the analysis and synthesis of matrix systems, (2006)

3. D. Kim, Mechatronics, Automation, Control, 1, 915, (2011)

4. V. Kulabuhov, XII Russian Conference on Control Problems VSPU-2014, pp. 438-448, (2014) 